\title{
Inbnaltşiuther|ingt.
}

Einleitung. Seite

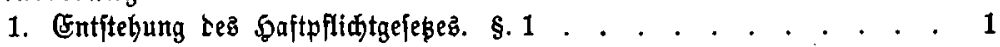

2. Inthalt und Saralter des Brejebes. \$. 2 . . . . . . . . . . . 6

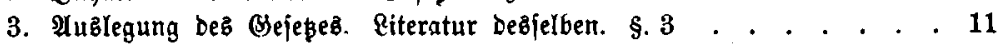

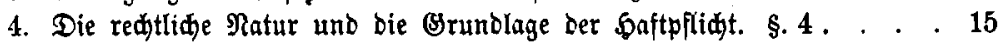

\section{Erläuterungen bes Gieję̧es̆.}

\section{Aite faftpfitht.}

\section{§. 1.}

A. Der Eifenbahnunternebmung . . . . . . . . . . . . . 23

1. Begriff Ler Eijenbahn . . . . . . . . . . . . . . . . $\mathbf{2 3}$

2. Die Boraubjegungen Der நaftpflidt . . . . . . . . . . . . 28

a) Bei bem Betriebe. . . . . . . . . . . . . . . . . . . 28

b) Ein $\mathbb{R e n}[$ L) . . . . . . . . . . . . . . . . . . 39

aa) getỏotet . . . . . . . . . . . . . . . . . . 40

bb) Lärperlia verlest . . . . . . . . . . . . . . . 41

c) Bemeiz Der Borauģęungen . . . . . . . . . . . . . 41

3. Der BetriebzunterneGmer . . . . . . . . . . . . . . . . 42

4. baftet für Den entitandenen Sdaden . . . . . . . . . . . . 46

5. Alusిnahme von ber நaftverbindlidfeit, wenn er beweift. . . . . . 50

a) Göhere semalt . . . . . . . . . . . . . . . 54

aa) Naturereigniffe . . . . . . . . . . . . . . 56

bb) Sandlungen Dritter . . . . . . . . . . . . . . 57

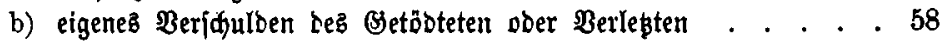

§. 2.

B. $\mathfrak{A n b e r e r ~ u n t e r n e g m u n g e n ~ . ~ . ~ . ~ . ~ . ~ . ~ . ~ . ~ . ~ . ~ . ~ . ~ . ~ . ~} 64$

1. Ber - betreift . . . . . . . . . . . . . . . . . . . 65

a) Bergmert. . . . . . . . . . . . . . . . . . . . 68

b) Steinbrud . . . . . . . . . . . . . . . . . 70

c) Bråberei . . . . . . . . . . . . . . . . . . . . . . . 70

d) Fabrif . . . . . . . . . . . . . . . . . . . . 71

2. Die Boraubjęungen Der ఏafttplidłt . . . . . . . . . . . . 73

a) Beridulden bejtimmter ßerjonen. . . . . . . . . . . . 74

aa) Bebollmådtigte . . . . . . . . . . . . . . . 74 
bb) SRepräfentanten

c) Bur Leitung oter Beauffidtigung Dez Betrieba angenom= mener ßerjonen . . . . . . . . . . . . . . . 78

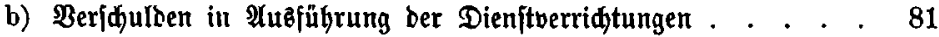

aa) Begriff bę Berfduldenz . . . . . . . . . . . . 81

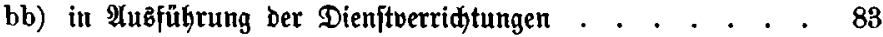

cc) als urjadbe ber Töbtung ober Rörperverlekung . . . . 84

3. Sgaft für ben baburd entítanbenen Gdjaben . . . . . . . . . 86

a) Rarafter ber \$aftberbindlidfeit . . . . . . . . . . . . . 86

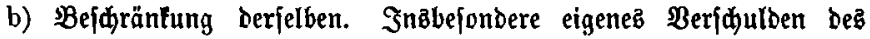
Betriebsiunternelymera . . . . . . . . . . . . . . . 87

4. Un Deffen Einfluß auf die Beltung dez §. 2 . . . . . . . . . . 89

\section{Der vermöge der foftpflidgt żu Ieiffende \$djadengerlał.}

\section{§. 3.}

A. Regelmäßiger umfang beźlelben . . . . . . . . . . . . . 93

1. Im Falle ber Töbtung . . . . . . . . . . . . . . . . 96

a) Die Rojten verfudter Geilung . . . . . . . . . . . . 96

b) Die Beerrigungbloiten . . . . . . . . . . . . . . . . . . 97

c) Der Bermögenżnadtheil Dez \&etöbteten Durd Störung Des Erwerbs 98

aa) währent ber Sranfgeit. . . . . . . . . . . . . 98

bb) Begriff Dez Grmerbz . . . . . . . . . . . . . . . . 99

cc) Bermögenżnadtheil . . . . . . . . . . . . . . 101

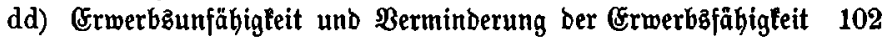

d) Crfą für entz̧ogene গ্llimente . . . . . . . . . . . . 105

aa) Boraublepung. . . . . . . . . . . . . . . . 105

1. zur 及eit bę నodę . . . . . . . . . . . . 105

2. vermöge Bsejęę begrüntete Unterhaltäverpflidtung bez Setöbteten . . . . . . . . . . . 106

bb) Die Erjabforderung. . . . . . . . . . . . . . 107

1. Rarafter Derjelben. . . . . . . . . . . . . 107

2. Umfang, infomeit iem Beredtigten in folge Deøె Todes: falla ber unterhalt entzogett worben ift. . . . . . 109

2. Im Falle ber ßörperverlegung . . . . . . . . . . . . . 114

a) Erfał ber Seilungakoiten . . . . . . . . . . . . . . 114

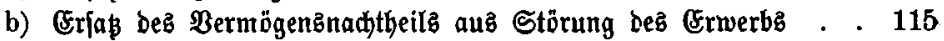

aa) Bermögenanadtheil. Folge Der Berlebung. Erwerbäunfähig: feit und Berminberung ber (Erwerbaffäbigleit . . . . . 115

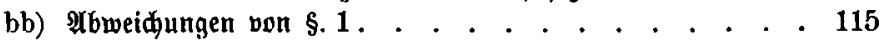

1. Der Berlebte . . . . . . . . . . . . . . 115

2. Zeitmeije und Lauernde (Grmerbsiunfăhigfeit ober Ber= minberung Der Erwerbęfäbiateit . . . . . . . . 116

cc) Bemeffung bez $\mathfrak{B e r m o ̈ g e n z ̇ n a d h t h e i l z ~ . ~ . ~ . ~ . ~ . ~ . ~ . ~ . ~ . ~} 116$

1. Unterfudung, ob unb weldhe (Erwerbafähigleit vorbant Den war . . . . . . . . . . . . 116 
2. ob in Folge ber Berlebung Erwerbâunfäbigteit ober Berminberung Der Erwerbafăhigfeit eingetreten ift . . 121

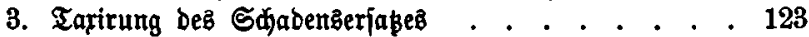

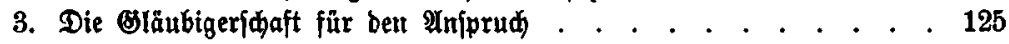

a) auf நeilungătoften . . . . . . . . . . . . . . . . 125

b) auf Beerbigungätoften . . . . . . . . . . . . . . . 126

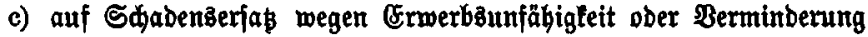
Der Erwerbaffăhigheit . . . . . . . . . . . . . . . 127

d) auf Sdabenserfał megen entzogenen unterbalta . . . . . . 127

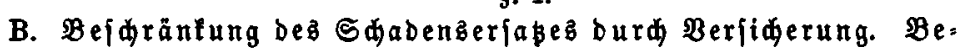
beutung berfelben im algemeinen. . . . . . . . . . . . 128

1. $\mathfrak{B o r a u s f e ̨ ̧ u n g e n . ~ . ~ . ~ . ~ . ~ . ~ . ~ . ~ . ~ . ~ . ~ . ~ . ~ . ~ . ~ . ~ . ~ . ~ . ~} 131$

a) Beftand eine Berjidjerung . . . . . . . . . . . . . 131

aa) Deå Getỏbteten ober ßerlestel . . . . . . . . . . 131

bb) gegen ben unfall . . . . . . . . . . . . . . . . . 132

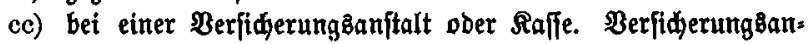
ftalt. Rnappidaftałaffe. Unterftüzungä: und Stantentafien.

ßenjionałaffen. . . . . . . . . . . . . . . . 136

b) Unter Mitleijtung bes Betriebsిınternehuterz . . . . . . . 136

aa) Begriff \er Mitleiftung . . . . . . . . . . . . 136

bb) Nidt unter einem Drittel Der Befammtleiftung . . . . 137

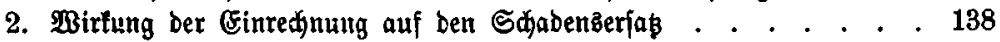

a) $\mathfrak{O b}$ nur bie bereitş auģgefübrte Reiftung einzurednen ift . . . 138

b) an ben Criagbereditigten . . . . . . . . . . . . . . 139

c) $\mathfrak{J}$ ít einzurednen . . . . . . . . . . . . . . . . . 141

d) auf bie Entidäbigung . . . . . . . . . . . . . . . . 142

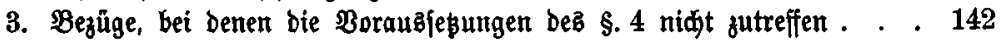

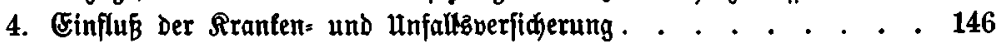

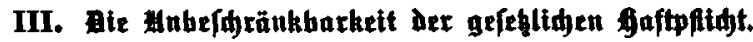

§. 5 .

Bebeutung bes §. 5 im Irthgemeinen . . . . . . . . . . . . . . 147

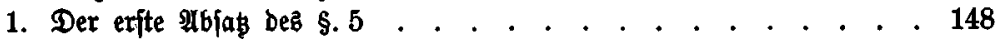

a) Die in ben $\$ \$ .1$ und 2 bezeidineten unternetgmen . . . . . 148

b) umfang bę \$erbotę . . . . . . . . . . . . . . . 148

c) Die Borte "z̧山 ihrem Bortheil" . . . . . . . . . . . . 148

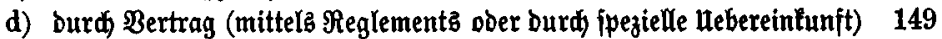

e) Iษ Boraนz . . . . . . . . . . . . . . . . . . 150

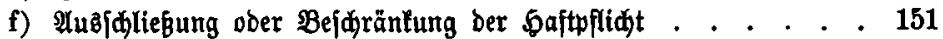

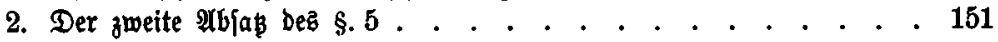

IV. Beltendmahung und Burekennung des Ghadeuserfahts.

A. Beltendataung . . . . . . . . . . . . . . . . . . 152

1. Strafredtlide, adminiftrative, aubergeridtlide . . . 152 
\$. 6.

2. Civilprozebuerfabren in Saftpflidtiaden . . . . . . . 158 3 wef bez $\$ .6$ im Allgemeinen . . . . . . . . . . . . . . . 158

1. Der erjte $\mathfrak{X b j a \}}$. . . . . . . . . . . . . . . . . . 159

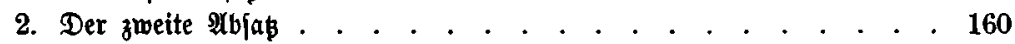

3. Der britte $\mathfrak{A b j a \mathfrak { s }}$. . . . . . . . . . . . . . . . . . 161

a) Îferlegung eines riđterliđen Gibez . . . . . . . . . . 161

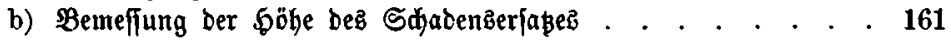

\section{\$. 7.}

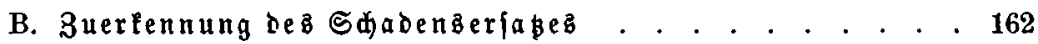

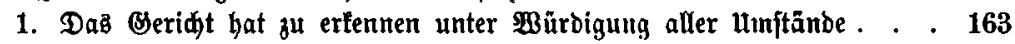

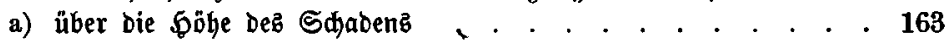

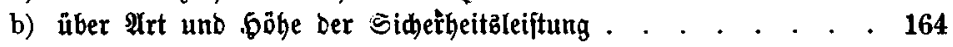

aa) Borauglepung. . . . . . . . . . . . . . . . 165

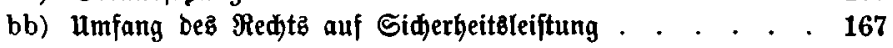

cc) ob aud bei anderer Feftitellung alsె Durd urtheil. . . . 167

dd) unter $\mathfrak{B u ̈ r b i g u r g}$ aller $\mathfrak{u}$ mftảnbe nad̆ freiem Ermeffen. . 168

1. ob eine Gidberheit zu bejtellen . . . . . . . . 168

2. in weldher $\mathfrak{A} r \mathrm{rt}$. . . . . . . . . . . . . . . 168

3. in welder bäbe . . . . . . . . . . . . . . 168

еe) $\mathfrak{A} \mathfrak{n}\{$ d)

2. 3uerfennung Des Erfąę fưr Den zutủnftigen unterhalt ober Ermerb 169

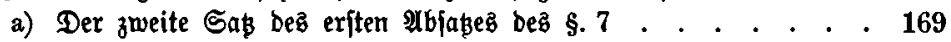

aа) 3uerfennung еinez Rapitalz........ . . . . . 170

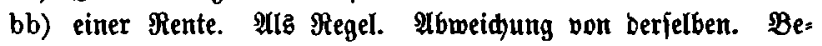
ftimmung über die 9 Irt ber 3ahlung . . . . . . . . . . 171

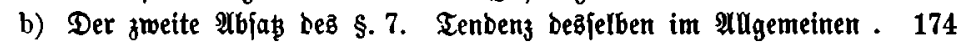

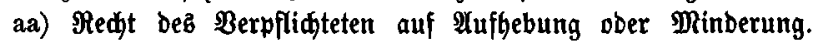
Borauajepung veränberter Berbältniffe . . . . . . . 177

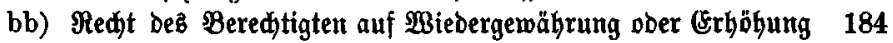

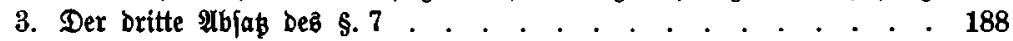

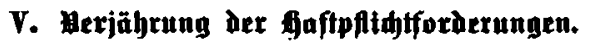

\$. 8.

Die Bebeutung beş $\$ .8$ im Arfgemeinen . . . . . . . . . . . . . 191

1. Berjāhrung ber Forderungen auf Sdhabenęerfaß̧ . . . . . . . 193

2. Berjährungäzeit, Dauer . . . . . . . . . . . . . . . . 196

a) Beginn Derjelbert. . . . . . . . . . . . . . . . . . . . . . 196

aa) in ber Regel vom Tage bez Unfallz . . . . . . . . . 196

bb) fửr bie Forberung wegen entzogenen unterbaltż vom Tage Deß Tobez̉ .. . . . . . . . . . . . . . . . . . . . . 196

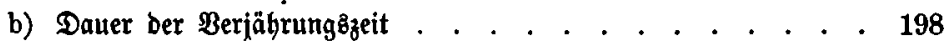

c) unterbredung. . . . . . . . . . . . . . . . . . 199

3. Wirkung . . . . . . . . . . . . . . . . . . . . . 201

a) $\mathfrak{I m}$ Allgemeinen. . . . . . . . . . . . . . . . . 201 
Eeite

b) Sm Einzelnen. $\mathfrak{B e r j a ̈ h ̆ r u n g ~ . ~ . ~ . ~ . ~ . ~ . ~ . ~ . ~ . ~ . ~ . ~ . ~ . ~} 202$

aa) ber Seilungktoften . . . . . . . . . . . . . . 203

bb) ber Beerbigungatojten . . . . . . . . . . . . . 203

cc) Dę Erjagez wegen verminberter ober verlorener Crwerbz= făbigleit. . . . . . . . . . . . . . . . . . 204

dd) Der Forberung Dę Unterhaltżbereditigten. . . . . . 205

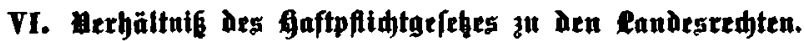
§. 9.

Bebeutung Deg $\$ .9$ im $\mathfrak{A l l g e m e i n e n ~ . ~ . ~ . ~ . ~ . ~ . ~ . ~ . ~ . ~ . ~ . ~ . ~ . ~ . ~} 206$

1. Der erjte $\mathfrak{A b j a \beta}$. . . . . . . . . . . . . . . . . . 208

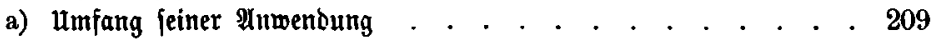

b) Boraublegungen. Ђaft . . . . . . . . . . . . . . . . 210

aa) beż unternehmerz einer $\mathfrak{A}$ nnlage . . . . . . . . . . 211

bb) Dder einer anberen ßerion . . . . . . . . . . . 213

c) anberührtbleiben ber Eanbez̆gejęe . . . . . . . . . . . 213

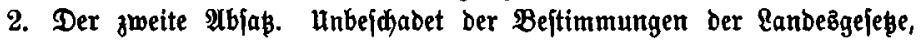
welde bem $\mathfrak{B}$ ejdăbigten einen höheren $\mathfrak{A}$ njprud gewähren . . . . 216

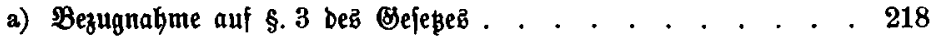

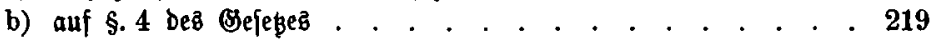

c) auf $\$ .6$ beşfelben . . . . . . . . . . . . . . . . 220

d) auf $\$ .8$ Deşelben . . . . . . . . . . . . . . . . 220

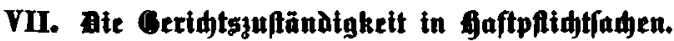

§. 10.

1. Muâlegung Dez \$. 10 . . . . . . . . . . . . . . . . 222

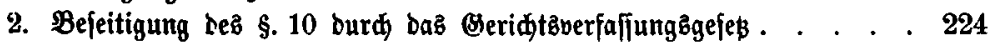


